\title{
Molecular characteristics of hepatocellular carcinomas from different age groups
}

\author{
Celina Ang1, Anthony Shields², Joanne Xiu, Zoran Gatalica ${ }^{4}$, Sandeep Reddy ${ }^{3}$, \\ Mohamed E. Salem ${ }^{5}$, Carol Farhangfar ${ }^{6}$, Jimmy Hwang 7 , Igor Astsaturov ${ }^{3,7}$ and \\ John L. Marshall ${ }^{5}$ \\ ${ }^{1}$ Department of Medicine, Hematology/Oncology, Icahn School of Medicine at Mount Sinai, New York, NY, USA \\ ${ }^{2}$ Department of Oncology, Molecular Imaging \& Diagnostics Program, Karmanos Cancer Center, Wayne State University, \\ Detroit, MI, USA \\ ${ }^{3}$ Department of Medical Affairs, Caris Life Sciences, Phoenix, AZ, USA \\ ${ }^{4}$ Department of Pathology, Caris Life Sciences, Phoenix, AZ, USA \\ ${ }^{5}$ Hematology/Oncology, Lombardi Comprehensive Cancer Center, Georgetown, University, Washington, DC, USA \\ ${ }^{6}$ Levine Cancer Institute, Carolinas Healthcare System, Charlotte, NC, USA \\ ${ }^{7}$ Department of Hematology/Oncology, Fox Chase Cancer Center, Philadelphia, PA, USA \\ Correspondence to: Celina Ang, email: celina.ang@mssm.edu \\ Keywords: hepatocellular carcinoma; age differences; multiplatform profiling; pathogenic mutations \\ Received: July 03, $2017 \quad$ Accepted: August 31, $2017 \quad$ Published: September 27, 2017 \\ Copyright: Ang et al. This is an open-access article distributed under the terms of the Creative Commons Attribution License 3.0 \\ (CC BY 3.0), which permits unrestricted use, distribution, and reproduction in any medium, provided the original author and source \\ are credited.
}

\section{ABSTRACT}

While most patients in Western countries who are diagnosed with HCC are in their 50 s and $60 \mathrm{~s}, \mathrm{HCC}$ diagnosed at extremes of the age spectrum (i.e., $<40$ years and $\geq 75$ years) are less common and have been linked with distinct geographic locations and etiologies. Using multiplatform profiling, we identified differences in genetic alterations and protein expression in different age groups within a large cohort of HCC patients $(\mathrm{N}=421)$. Young adult HCC patients (18-39 years' old) were more likely to be female, living in the West and Midwestern United States, and showed decreased androgen receptor, drug resistance and pro-angiogenic protein expression compared to older patients. TP53 mutations were the most frequent alteration in young adults (19\%), whereas CTNNB1 mutations occurred in $30-33 \%$ of patients $\geq 40$ years' old. The overall frequency of pathogenic and presumed pathogenic mutations was observed to increase significantly with advancing age. To our knowledge, these data represent one of the only studies to analyze age-specific molecular profiles in HCC, and provide a basis for further exploration and validation of these findings with respect to their clinical and therapeutic implications.

\section{INTRODUCTION}

Hepatocellular carcinoma (HCC) is an aggressive malignancy, representing the second and sixth leading cause of cancer deaths worldwide in men and women, respectively [1]. HCC typically arises in the context of chronic liver disease and cirrhosis due to viral hepatitis, excessive alcohol consumption, fatty liver disease and other risk factors. While liver transplantation and resection offer the possibility of long-term survival, advanced HCC is uniformly lethal and there are limited treatment options.
The majority of HCC patients in the Western hemisphere are diagnosed in their 50s and 60s, though HCCs diagnosed at extremes of the age spectrum (i.e., < 40 years, $\geq 75$ years) also occur. The highest incidence of HCC in individuals $\geq 75$ years' old, particularly in men, has been reported in low-risk Western countries, South Africa and Egypt where hepatitis $\mathrm{C}$ prevails [2]; whereas young adult patients (i.e., 20-40 years' old) diagnosed with HCC primarily reside in hepatitis $\mathrm{B}$ endemic regions. In one study, over $85 \%$ of patients $\leq 40$ years of age were found to be hepatitis B surface antigen seropositive compared to $60 \%$ 
of patients $>40$ years $(\mathrm{p}=0.003)$, underscoring the strong association between hepatitis B and young onset HCC [3]. East Asian countries have an HCC prevalence in young adults ranging from $0.9-10.9 \%$ [3]. Among African Blacks with hepatitis B, $43 \%$ are diagnosed with HCC before 40 years of age [4]. Apart from etiologic and geographic differences, younger individuals with HCC tend to present with a higher serum $\alpha$-fetoprotein and more advanced stage at diagnosis [3, 5-8]. In contrast, $\mathrm{HCC}$ in the elderly has been linked with female sex, chronic hepatitis $\mathrm{C}$ or no history of viral hepatitis, and a higher prevalence of normal liver than younger patients [9]. Comparisons of age-specific differences in survival have yielded conflicting results with some studies reporting poorer outcomes in younger vs older patients and others reporting no difference $[3,6,10,11-15]$. To our knowledge, there have not been any studies that have evaluated molecular differences in HCCs between young and elderly patients. We sought to ascertain differences in molecular profiles of $\mathrm{HCC}$ patients across the age spectrum and their potential therapeutic implications.

\section{RESULTS}

\section{Demographics}

A total of 421 specimens were included in this dataset: $39(9 \%)$ were from young adults (18-39 years' old), 46 (11\%) from elderly patients ( $\geq 75$ years' old) and $336(80 \%)$ from the intermediate age group (40-74 years' old). There was a significantly higher proportion of females in the young adult HCC subgroup (54\%) compared to intermediate age $(23 \%, \mathrm{p}<0.0001)$ and elderly patients $(33 \%, \mathrm{p}=0.048)$. HCC metastatic to another organ comprised $38 \%, 30 \%$ and $20 \%$ of the young adult, intermediate age and elderly cohorts, respectively, but these differences were not significant. The proportion of HCC metastases by age group is illustrated in Figure 1 and the breakdown of metastatic sites is shown in Table 1. The most frequently sampled metastatic sites were lymph nodes in young adults and the lungs in the intermediate age group. There was no significant difference among the groups with regards to the use of primary or metastatic tumor for these analyses. Therefore, the subsequent comparisons performed on the tumor groups stratified by age consisted of both primary and metastatic tumors.

Information on geographic region of residence was available on 398 patients. There were 12 patients from Israel, France, China, Russia, Lebanon, the United Kingdom, Australia and Belgium. Amongst patients living in the United States, the majority resided in the Southeast (29\%) followed by the Northeast (25\%), West $(22 \%)$, Midwest and Southwest (12\% each). Elderly patients were more prevalent than younger patients in the Southeast and Southwest, whereas patients $<75$ years' old were more prevalent in the West and Midwest than elderly patients.
The breakdown of region of residence by age amongst patients living in the United States is illustrated in Figure 2.

\section{Protein expression}

As shown in Figure 3, androgen receptor (AR) expression was significantly less frequent in young adults $(2 / 33,6 \%)$ compared to the elderly $(10 / 38,26 \%$, $\mathrm{p}=0.026)$ and intermediate age groups $(71 / 251,28 \%$, $\mathrm{p}=0.007)$. Focusing on male patients, AR expression was significantly less common in young adults $(1 / 16,6 \%)$ than in the intermediate age $(67 / 193,35 \%, \mathrm{p}=0.019)$ and elderly $(8 / 25,32 \%, p=0.052)$ groups. In females, there was also a trend towards increased AR expression with advancing age: young adults $(1 / 17,6 \%)$, intermediate age $(4 / 58,7 \%)$ and elderly patients $(2 / 13,15 \%, \mathrm{p}>0.05)$.

MGMT expression was significantly more common in intermediate age than young adult patients $[71 \%$ $(190 / 267)$ vs 49\% (17/35), $\mathrm{p}=0.007]$, and was also more frequent in the elderly subgroup $(26 / 39,67 \%)$. There were significant differences in MRP-1 expression in HCCs in young adults $(6 / 10,60 \%)$ compared to intermediate age $(80 / 93,86 \%, \mathrm{p}=0.035)$ and elderly patients $(18 / 19$, $95 \%, \mathrm{p}=0.019)$. SPARC expression was absent in young adult HCCs but was present in $12 \%(25 / 214)$ and $13 \%(4 / 31)$ of tumors from intermediate age and elderly patients, respectively. PDGFR and PD-L1 were expressed in intermediate age $[14 \%(5 / 36)$ and $10 \%$ $(7 / 68)$, respectively] and elderly patients $[29 \%(2 / 7)$ and $17 \%(1 / 6)$, respectively], but not young adults $[0 \%(0 / 7)$ and $0 \%(0 / 7)$, respectively]. The hepatocyte growth factor receptor c-Met was expressed in over $25 \%$ of patients $\geq 40$ years' old compared to only $9 \%$ of young adults. None of these differences were statistically significant.

\section{Next generation sequencing}

A total of 47 genes were analyzed for wellcharacterized hotspots and their respective alteration frequencies and distribution across the different age groups are shown in Figure 4. Differences in the frequency of pathogenic and presumed pathogenic variants are highlighted in Table 2. TP53 mutations were the most common alteration observed in young adults, but their frequency did not differ significantly amongst the age groups. CTNNB1 mutations were significantly less frequent in young adults $(2 / 21,10 \%)$ than in intermediate age $(44 / 145,30 \%, p=0.046)$ and elderly $(5 / 15,33 \%$, $\mathrm{p}>0.050)$ patients. Elderly patients exhibited a higher frequency of alterations in PIK3CA/Akt/mTOR pathway components PIK3CA, PTEN and PTPN11 (7-13\%) compared to intermediate age and young adult patients $(0-$ $1 \%)$. These differences were not significant, though the proportion of elderly and young adult patients tested was much smaller than the intermediate age cohort. Alterations in JAK3, ATM, ERBB4, KRAS and NRAS were also present and did not differ statistically amongst the groups. 
Table 1: Sites of HCC metastases in different age groups

\begin{tabular}{lccc}
\hline Metastatic site & Young adult $(\mathbf{N}=\mathbf{1 5})$ & Intermediate age $(\mathbf{N}=\mathbf{1 0 2})$ & Elderly $(\mathbf{N}=\mathbf{9})$ \\
\hline Lymph nodes & 6 & 13 & 2 \\
Lung & 4 & 23 & 1 \\
Peritoneum & 2 & 8 & 2 \\
Bone & 2 & 17 & 0 \\
Connective tissue & 1 & 18 & 2 \\
Brain & 0 & 4 & 0 \\
Other & 0 & 19 & 2 \\
\hline
\end{tabular}

The overall frequency of alterations appeared to increase with age. Focusing only on pathogenic and presumed pathogenic alterations, the frequency was significantly lower in young adults (0.38 mutations/ case) versus intermediate age $(0.71, \mathrm{p}=0.012)$ and elderly patients $(0.93, \mathrm{p}=0.038)$.

\section{Biomarker expression in primary and metastatic sites}

Differences in biomarker expression were noted between primary and metastatic HCC sites as shown in Table 3. Across all age groups, p-glycoprotein expression was significantly more frequent in primary $\mathrm{HCC}$ than in metastases, whereas TOP2A, thymidylate synthase and TUBB3 expression were more frequent in metastases. There was a significantly higher occurrence of PTEN mutations in metastases than in primary HCCs, but the absolute frequencies were low (3.6\% and $0 \%$, respectively). Among the three subgroups, only the intermediate age group demonstrated significant differences in biomarkers between primary and metastatic HCC. The same expression patterns seen in the entire study population were also observed in this subgroup, except that there was a higher incidence of $P I K 3 C A$ mutations in metastases $(4.5 \%)$ than in primary $\mathrm{HCC}(0 \%)$.

\section{DISCUSSION}

In this retrospective series, differences in demographic and molecular features of HCC patients across different age groups were detected. We observed

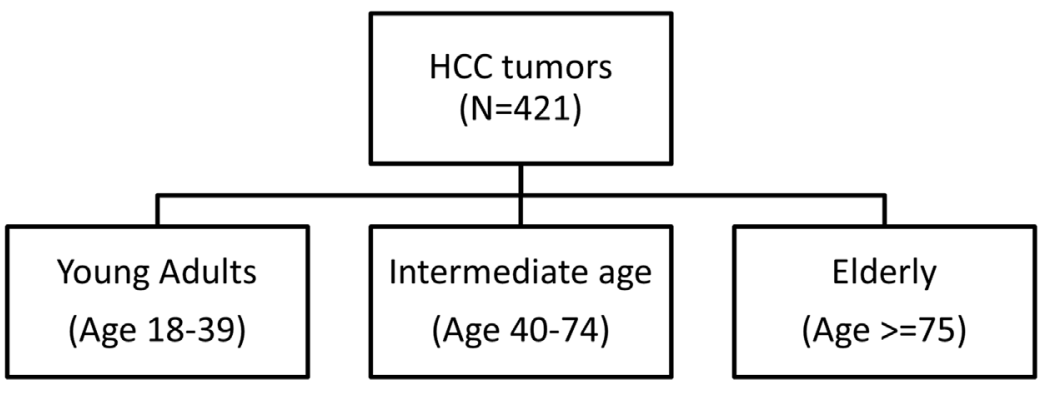

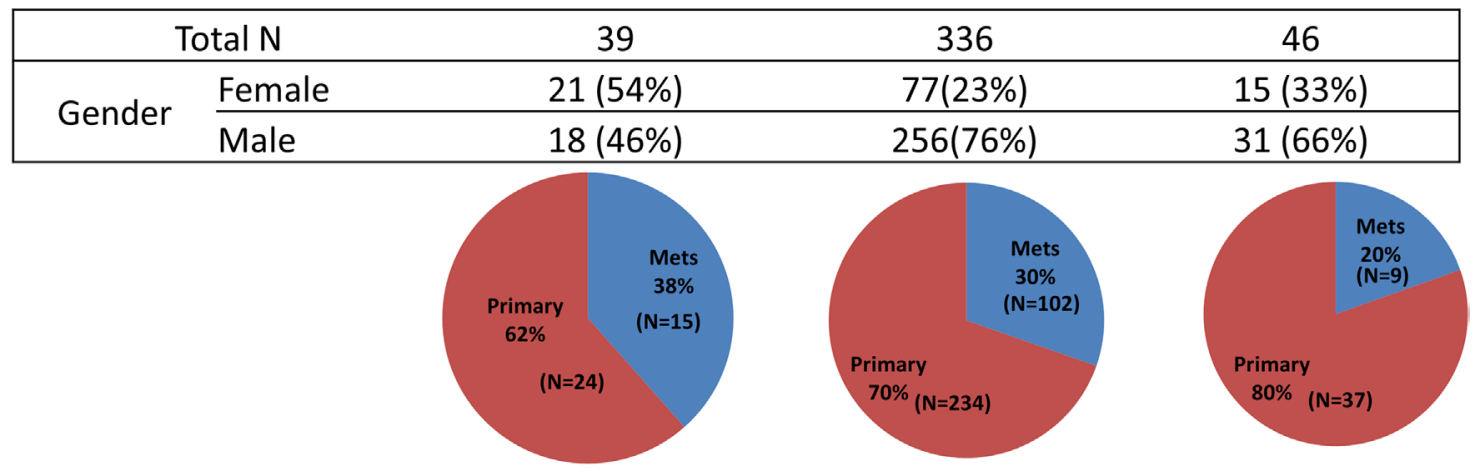

Figure 1: Study cohort composition. 
that young adults were more frequently female, residing in Western and Midwestern states, and less frequently expressed the AR, drug resistance and pro-angiogenic proteins compared to older patients. CTNNB1 mutations, one of the most common pathogenic alterations in $\mathrm{HCC}$, were significantly less common in young adults than in patients 40 years and older. Elderly HCC patients exhibited increased PI3K/Akt/mTOR pathway alterations compared to younger patients.
From a therapeutic perspective, these data suggest the potential for differential sensitivities amongst the subgroups to various agents. Increased MGMT and SPARC expression in patients $\geq 40$ years' old suggests that they might be less responsive to alkylating agents, but more sensitive to taxanes or fluoropyrimidines. SPARC overexpression has been shown to resensitize HepG2 cells to 5-fluorouracil [16]. Intermediate age and elderly patients might also be susceptible to AR inhibitors. The

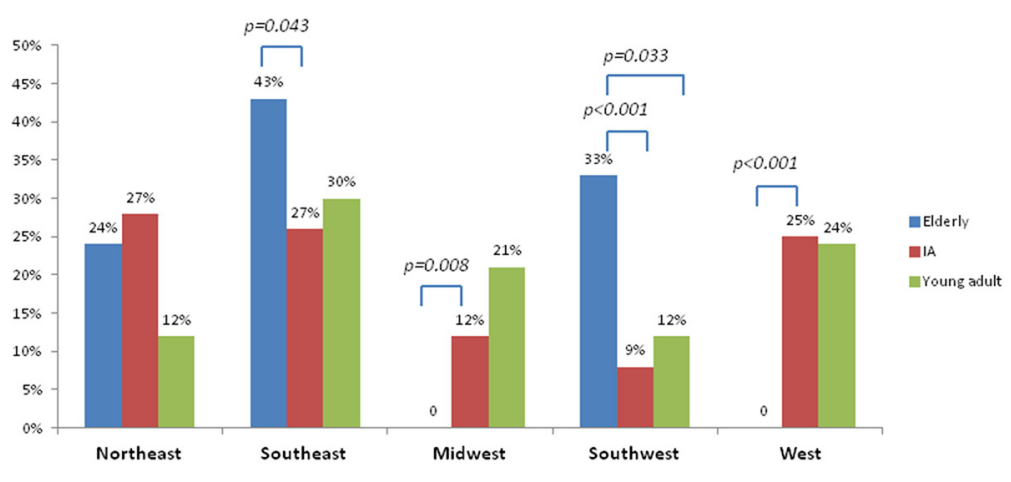

Figure 2: Breakdown of United States region of residence by age.

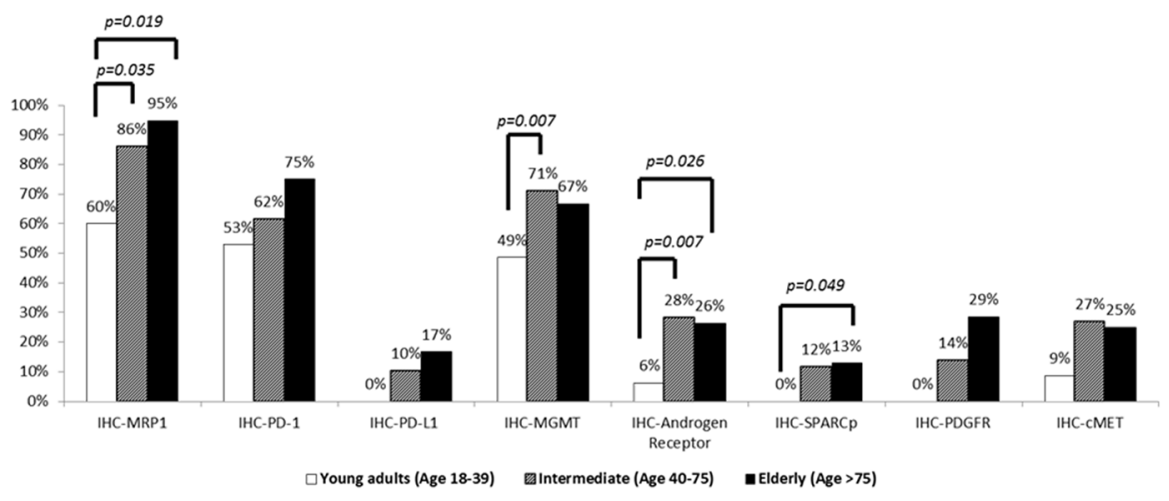

Figure 3: Comparison of protein expression by immunohistochemistry across age groups.

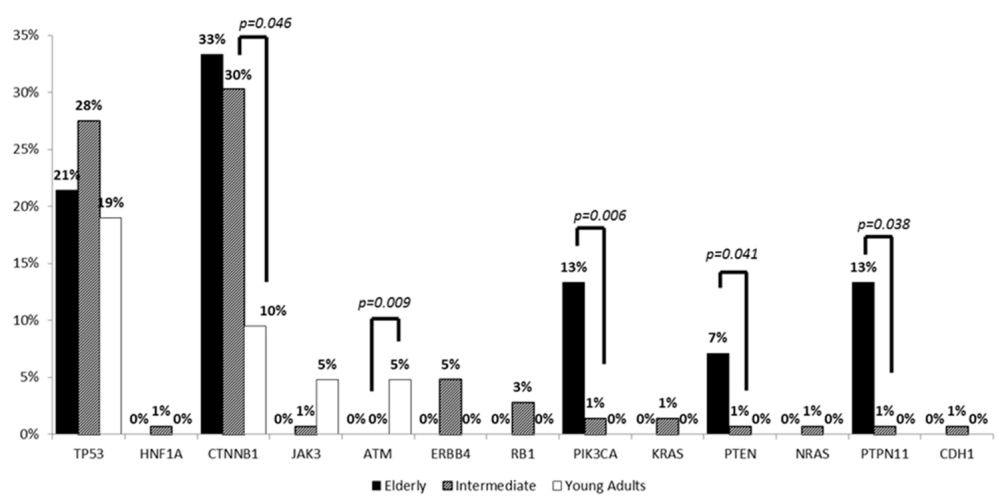

Figure 4: Comparison of pathogenic and presumed pathogenic alterations across the age groups. 
Table 2: Frequencies of pathogenic and presumed pathogenic alterations in different age groups

\begin{tabular}{lccccccccc}
\hline & $\begin{array}{c}\text { Young Adult } \\
(\mathbf{1 8 - 3 9} \text { years })\end{array}$ & $\begin{array}{c}\text { Intermediate age } \\
(\mathbf{4 0 - 7 4} \text { years })\end{array}$ & $\begin{array}{c}\text { Elderly } \\
(\geq \mathbf{7 5} \text { years })\end{array}$ & YA vs IA & YA vs E & IA vs E \\
\hline N+/N total & $\mathbf{\%}$ & $\mathbf{N}+/ \mathbf{N}$ total & $\mathbf{\%}$ & $\mathbf{N}+/ \mathbf{N}$ total & $\mathbf{\%}$ & $\mathbf{p}$-value & $\mathbf{p}$-value & p-value \\
\hline TP53 & $4 / 21$ & 19 & $39 / 142$ & 28 & $3 / 14$ & 21 & NS & NS & NS \\
HNF1A & $0 / 19$ & 0 & $1 / 136$ & 0.7 & $0 / 13$ & 0 & NS & NS & NS \\
CTNNB1 & $2 / 21$ & 10 & $44 / 145$ & 30 & $5 / 15$ & 33 & 0.046 & NS & NS \\
JAK3 & $1 / 21$ & 5 & $1 / 146$ & 1 & $0 / 15$ & 0 & NS & NS & NS \\
ATM & $1 / 21$ & 5 & $0 / 144$ & 0 & $0 / 15$ & 0 & 0.008 & NS & NS \\
ERBB4 & $0 / 21$ & 0 & $7 / 145$ & 5 & $0 / 15$ & 0 & NS & NS & NS \\
RB1 & $0 / 21$ & 0 & $4 / 143$ & 3 & $0 / 15$ & 0 & NS & NS & NS \\
PIK3CA & $0 / 21$ & 0 & $2 / 141$ & 1 & $2 / 15$ & 13 & NS & NS & 0.006 \\
KRAS & $0 / 21$ & 0 & $2 / 146$ & 1 & $0 / 15$ & 0 & NS & NS & NS \\
PTEN & $0 / 21$ & 0 & $1 / 142$ & 1 & $1 / 14$ & 7 & NS & NS & 0.041 \\
NRAS & $0 / 21$ & 0 & $1 / 144$ & 1 & $0 / 15$ & 0 & NS & NS & NS \\
PTPN11 & $0 / 21$ & 0 & $1 / 146$ & 1 & $1 / 15$ & 13 & NS & NS & 0.038 \\
CDH1 & $0 / 21$ & 0 & $1 / 146$ & 1 & $0 / 15$ & 0 & NS & NS & NS \\
\hline
\end{tabular}

$\mathrm{YA}=$ young adult; $\mathrm{IA}=$ intermediate age $\mathrm{E}=$ elderly; $\mathrm{NS}=$ not significant

higher incidence of $\mathrm{PI} 3 \mathrm{~K} / \mathrm{Akt} / \mathrm{mTOR}$ pathway mutations in elderly patients may predict greater sensitivity to agents targeting this cascade compared to younger patients. However, the increased expression of MRP-1 in patients $\geq 40$ years' old may represent an adaptive response to a greater lifetime cumulative exposure to xenobiotics [17] that renders their disease more resistant to treatment than in their younger counterparts.

Differences in therapeutic sensitivities may also exist between primary tumors and metastases. The frequency of p-glycoprotein expression observed in primary HCCs is compatible with the existing literature [18] and is thought to underlie the resistance of these tumors to chemotherapy. Increased expression of TOP2A, thymidylate synthase, TUBB3 and mutations in PIK3CA and PTEN in HCC metastases suggest that they may be less responsive to 5-fluorouracil and taxanes, but more sensitive to anthracyclines and mTOR inhibitors. Although the predictive clinical value of these biomarkers is limited as cytotoxic chemotherapy is not routinely used to treat HCC, and the mTOR inhibitor everolimus was not active in a phase III trial [19], the different expression patterns nevertheless support the hypothesis that there may be agespecific and tumor site-specific molecular differences in HCC.

We observed a statistically significant increase in the frequency of pathogenic and presumed pathogenic mutations with increasing age. This is different from tumor mutation load (TML) which is thought to correlate with immunogenicity. TML values calculated from somatic missense mutations using the 592 gene panel were unavailable for vast majority of the cases investigated in the current analysis. From a separate study with 98 tumors sequenced with 592-gene panel, we observed TML values to be 5.5 mutations/megabase in the young adults group $(\mathrm{n}=6), 7.6$ in the intermediate group $(\mathrm{n}=73)$ and 6.7 in the elderly group $(n=18)$, with no statistical significant differences seen amongst the groups. Given recent evidence of nivolumab activity in HCC [20], the relationship between TML, PD-L1 expression and response to checkpoint inhibitors is of interest. PD-L1 expression was assessed in some tumors and was found to be absent in young adults. Testing in a larger proportion of patients from extremes of the age-spectrum will be necessary to determine if there are age-specific differences in PD-L1 expression.

Our results also suggest etiologic associations and demographic features of young adult and older HCC patients that are compatible with the existing literature. Increased MGMT expression in patients $\geq 40$ years' old may reflect lower $M G M T$ methylation levels which have been linked with hepatitis $\mathrm{C}$ and non-alcohol associated HCC [21]. The higher incidence of CTNNB1 mutations in this age group also suggests that they are less likely to have hepatitis B induced HCC [22]. However, increasing tumor AR expression with advancing age may 
Table 3: Biomarker expression and tumor genotype in primary $\mathrm{HCC}$ and metastatic sites

\begin{tabular}{|c|c|c|c|c|}
\hline & Significant Biomarkers & Mets. Vs. Primary & Percent & p values \\
\hline \multirow{5}{*}{ Entire study group } & IHC-p-glycoprotein & $61 / 93$ vs. $155 / 199$ & $66 \%$ vs. $78 \%$ & 0.029 \\
\hline & IHC-TOP2A & $52 / 99$ vs. $96 / 248$ & $52 \%$ vs. $39 \%$ & 0.016 \\
\hline & IHC-thymidylate synthase & $47 / 146$ vs. $61 / 254$ & $32 \%$ vs. $24 \%$ & $<0.001$ \\
\hline & IHC-TUBB3 & $11 / 69$ vs. $11 / 151$ & $16 \%$ vs. $7.3 \%$ & 0.045 \\
\hline & NGS-PTEN & $2 / 55$ vs. $0 / 123$ & $3.6 \%$ vs. 0 & 0.032 \\
\hline \multirow[t]{3}{*}{ Young adults } & & None & & \\
\hline & IHC-p-glycoprotein & $49 / 73$ vs. $121 / 152$ & $67 \%$ vs. $80 \%$ & 0.048 \\
\hline & IHC-TOP2A & $44 / 79$ vs. $74 / 195$ & $56 \%$ vs. $38 \%$ & 0.006 \\
\hline \multirow[t]{3}{*}{ Intermediate age } & IHC-thymidylate synthase & 39/86 vs. $45 / 199$ & $45 \%$ vs. $3 \%$ & $<0.001$ \\
\hline & IHC-TUBB3 & $10 / 58$ vs. $8 / 177$ & $17 \%$ vs. $4.5 \%$ & 0.031 \\
\hline & NGS-PIK3CA & 2/44 vs. $0 / 99$ & $4.5 \%$ vs. 0 & 0.032 \\
\hline Elderly & & None & & \\
\hline
\end{tabular}

indicate a link with the hepatitis B virus given evidence of a positive feedback loop with AR signaling [23]. The higher frequency of PI3K/Akt/mTOR pathway mutations in elderly patients suggests that they are more likely to be of Western than Asian origin [24]. In contrast, TP53 mutations - the most common alteration observed in young adults - are more frequent in Asian than in Western populations, and are strongly associated with aflatoxin exposure and hepatitis B [22, 24]. Differences in the variables that drive hepatocarcinogenesis in different age groups, and their temporal and spatial interplay with AR signaling, viral status and sex remain to be elucidated.

While prior studies have reported that elderly HCC patients are more likely to be female, possibly as a result of the longer life expectancy of women $[9,12$, $13,15]$, a decreased female prevalence in patients $\geq 75$ years was observed in this cohort. Since many of the studies reporting an increased female to male ratio among elderly HCC patients were conducted in East Asia, the discrepant findings of our study may be partly explained by geographic and racial or ethnic differences. Due to the unavailability of racial and ethnic information, we are unable to determine whether sex ratio differs by race and ethnicity within our Western study cohort.

Weaknesses of this study include the absence of clinical, race or ethnicity data which prevents us from confirming or refuting our assumptions about disease etiology based on the molecular findings, as well as commenting on differences in disease characteristics and oncologic outcomes between the age groups. In addition, the proportion of young adult and elderly patients is small compared to the intermediate age group which encompasses a broad and heterogeneous age range. These differences might have masked other molecular differences between and within the groups. The small number of cases analyzed for some biomarkers also limits the robustness of the comparisons and the conclusions that can be drawn. Given the absence of information on patients' treatment histories and the timing of specimen collection in relation to specific agents or modalities, we are unable to account for the potential confounding effect of therapy on biomarker expression. Furthermore, the panel of genes tested is not an exhaustive list of all known contributors to hepatocarcinogenesis and maintenance; for example, genetic aberrations affecting chromatin remodeling, telomere maintenance and oxidative stress [25] were not included. Rather, the genes included were selected for their known prognostic and/or therapeutic significance.

In summary, multiplatform profiling data reveals molecular distinctions between young adult and older HCC patients, highlighting potential differences in therapeutic targets amongst the groups. To our knowledge, these data represent one of the only studies to analyze agespecific molecular profiles in HCC, and provide a basis for further exploration and validation of these findings with respect to their clinical and therapeutic implications.

\section{MATERIALS AND METHODS}

Gene sequencing, amplification and protein expression data from anonymized HCC specimens submitted to Caris Life Sciences between 2009 and March of 2016 were retrospectively reviewed. H\&E slides were prepared for each tumor sample and were reviewed by board-certified pathologists to verify the diagnosis of $\mathrm{HCC}$ on the pathology reports accompanying the tumor samples. Fibrolamellar liver carcinoma, hepatoblastoma and hepatosarcoma were excluded. Not all the same tests were performed on all tumors investigated, due to the 
different tests requested by treating physicians for each patient, or due to the advancement of testing technologies over time. For NextGen sequencing, tumors tested in 2015 or earlier were sequenced with MiSeq platform while those tested in 2016 or later were sequenced with NextSeq platform. Because patients consented to tumor specimen submission and molecular profiling but not access to their medical records, only basic demographic information was available. Patients were stratified into young adult (1839 years' old), intermediate age (40-74 years' old) and elderly ( $\geq 75$ years' old) subgroups. For patients living in the United States, region of residence was determined by zip code. The Chi-square test was used for statistical comparisons.

Immunohistochemistry (IHC) was performed on 408 tumors on full formalin-fixed paraffin-embedded (FFPE) sections on glass slides. Slides were stained using an automated system (Benchmark, Ventana Medical Systems, Tucson, AZ; Autostainer, DAKO, Carpinteria, CA) as per the manufacturer's instructions, and were optimized and validated per CLIA/CAO and ISO requirements. Tumor cells were scored for all proteins of interest with the exception of PD-1, which was scored on tumor infiltrating lymphocytes. Staining was scored for intensity $(0=$ no staining; $1+=$ weak staining; $2+=$ moderate staining; $3+$ $=$ strong staining) and percent of cells staining positive for the protein $(0-100 \%)$. The primary antibody used against PD-L1 was SP142 (Spring Biosciences). The staining was regarded as positive if its intensity on the membrane of the tumor cells was $>=2+$ and the percentage of positively stained cells was $>5 \%$. Results were categorized as positive or negative by defined thresholds specific to each marker, based on published clinical literature that associates biomarker status with patient responses to therapeutic agents. A board-certified pathologist evaluated all IHC results independently.

Next-generation sequencing (NGS) was performed on 182 tumors on genomic DNA isolated from FFPE tumor samples using either the MiSeq $(\mathrm{N}=154)$ platform or the NextSeq $(\mathrm{N}=28)$ platform (Illumina, Inc., San Diego, CA). No matched normal tissue was sequenced. For tumors tested with MiSeq, specific regions of the genome were amplified using the Illumina TruSeq Amplicon Cancer Hotspot panel. For tumors tested with NextSeq, a customdesigned SureSelect XT assay was used to enrich 592 whole-gene targets (Agilent Technologies, Santa Clara, CA). All variants were detected with $>99 \%$ confidence based on allele frequency and amplicon coverage with an average sequencing depth of coverage of $>500$ and with an analytic sensitivity of $5 \%$. Genetic variants identified were interpreted by board-certified molecular geneticists and categorized as 'pathogenic,' 'presumed pathogenic,' 'variant of unknown significance,' 'presumed benign,' or 'benign,' according to the American College of Medical Genetics and Genomics (ACMG) standards. When assessing mutation frequencies of individual genes, 'pathogenic,' and 'presumed pathogenic, were counted as mutations while 'benign' or 'presumed benign' variants and 'variants of unknown significance' were excluded.

\section{Author contributions}

CA and JX oversaw the study design and were responsible for data analysis and manuscript writing. All authors reviewed and approved the manuscript.

\section{ACKNOWLEDGMENTS}

This work was presented at the 2016 European Society of Medical Oncology conference in Copenhagen, Denmark.

\section{CONFLICTS OF INTEREST}

The authors declare no conflicts of interest.

\section{REFERENCES}

1. Torre LA, Bray F, Siegel RL, Ferlay J, Lortet-Tieulent J, Jemal A. Global cancer statistics, 2012. CA Cancer J Clin. 2015; 65:87-108.

2. Nordenstedt H, White DL, El-Serag HB. The changing pattern of epidemiology in hepatocellular carcinoma. Dig Liver Dis. 2010; 42:S206-214.

3. Chang PE, Ong WC, Lui HF, Tan CK. Is the prognosis of young patients with hepatocellular carcinoma poorer than the prognosis of older patients? A comparative analysis of clinical characteristics, prognostic features, and survival outcome. J Gastroenterol. 2008; 881-8

4. Yang JD, Gyedu A, Afihene MY, Duduyemi BM, Micah E, Kingham TP, Nyirenda M, Nkansah AA, Bandoh S, Duguru MJ, Okeke EN, Kouakou-Lohoues MJ, Abdo A, et al. Hepatocellular carcinoma occurs at an earlier age in Africans, particularly in association with chronic hepatitis B. Am J Gastroenterol. 2015; 110:1629-31.

5. Aramaki M, Kawano K, Sasaki A, Ohno T, Tahara K, Kai $\mathrm{S}$, Iwashita Y, Kitano S. Hepatocellular carcinoma in young adults. Hepatogastroenterology. 2005; 52:1795-7.

6. Trevisani F, D'Intino PE, Grazi GL, Caraceni P, Gasbarrini A, Colantoni A, Stefanini GF, Mazziotti A, Gozzetti G, Gasbarrini G, Bernardi M. Clinical and pathologic features of hepatocellular carcinoma in young and older Italian patients. Cancer. 1996; 77:2223-32.

7. Yamazaki Y, Kakizaki S, Sohara N, Sato K, Takagi H, Arai H, Abe T, Katakai K, Kojima A, Matsuzaki Y, Mori M. Hepatocellular carcinoma in young adults: the clinical characteristics, prognosis and findings of a patient survival analysis. Dig Dis Sci. 2007; 52:1103-7.

8. Chen CH, Chang TT, Cheng KS, Su WW, Yang SS, Lin HH, Wu SS, Lee CM, Changchien CS, Chen CJ, Sheu JC, Chen 
DS, Lu SN. Do young hepatocellular carcinoma patients have worse prognosis? The paradox of age as a prognostic factor in the survival of hepatocellular carcinoma patients. Liver Int. 2006; 26:766-773.

9. Nishikawa H, Kimura T, Kita R, Osaki Y. Treatment for hepatocellular carcinoma in elderly patients: a literature review. J Cancer. 2013; 4:635-43.

10. Hung AK, Guy J. Hepatocellular carcinoma in the elderly: meta-analysis and systematic literature review. World J Gastroenterol. 2015; 21:12197-210.

11. Cho SJ, Yoon JH, Hwang SS, Lee HS. Do young hepatocellular carcinoma patients with relatively good liver function have poorer outcomes than elderly patients? J Gastroenterol Hepatol. 2007; 22:1226-31.

12. Takahashi H, Mizuta T, Kawazoe S, Eguchi Y, Kawaguchi Y, Otuka T, Oeda S, Ario K, Iwane S, Akiyama T, Ozaki I, Fujimoto K. Efficacy and safety of radiofrequency ablation for elderly hepatocellular carcinoma patients. Hepatol Res. 2010;40:997-1005.

13. Kao WY, Chiou YY, Hung HH, Su CW, Chou YH, Huo TI, Huang YH, Wu WC, Lin HC, Lee SD, Wu JC. Younger hepatocellular carcinoma patients have a better prognosis after percutaneous radiofrequency ablation therapy. J Clin Gastroenterol. 2012;46:62-70.

14. Su CW, Lei HJ, Chau GY, Hung HH, Wu JC, Hsia CY, Lui WY, Su YH, Wu CS, Lee SD. The effect of age on the longterm prognosis of patients with hepatocellular carcinoma after resection surgerY: a propensity score matching analysis. Arch Surg. 2012;147:137-44.

15. Reddy SK, Barbas AS, Turley RS, Gamblin TC, Geller DA, Marsh JW, Tsung A, Clary BM, Lagoo-Deenadyalan S. Major liver resection in elderly patients: a multiinstitutional analysis. J Am Coll Surg. 2011;212:787-95.

16. Hua HW, Jiang F, Hung Q, Liao ZJ, Ding G. Re-sensitization of 5-FU resistance by SPARC through negative regulation of glucose metabolism in hepatocellular carcinoma. Tumour Biol. 2015; 36:303-13.
17. Namasaki T, Schaeffeler E, Fukui H, Yoshiji H, Nakajima Y, Fritz P, Schwab M, Nies AT. Differential expression of drug uptake and efflux transporters in Japanese patients with hepatocellular carcinoma. Drug Metab Dispos. 2014; 42:2033-40

18. $\mathrm{Ng} \mathrm{IO}$, Liu CL, Fan ST, Ng M. Expression of p-glycoprotein in hepatocellular carcinoma. A determinant of chemotherapy response. Am J Clin Pathol. 2000; 113:355-63.

19. Zhu AX, Kudo M, Assenat E, Cattan S, Kang YK, Lim HY, Poon RT, Blanc JF, Vogel A, Chen CL, Dorval E, PeckRadosavlijevic M, Santoro A, et al. Effect of everolimus on survival in advanced hepatocellular carcinoma after failure of sorafenib: the EVOLVE-1 randomized clinical trial. JAMA. 2014;312:57-67.

20. El-Khoueiry AB, Sangro B, Yau T, Crocenzi TS, Kudo M, Hsu C, Kim TY, Choo SP, Trojan J, Welling TH Rd, Meyer T, Kang YK, Yeo W, et al. Nivolumab in patients with advanced hepatocellular carcinoma (CheckMate 040): an open-label, non-comparative, phase $1 / 2$ dose escalation and expansion trial. Lancet. 2017.

21. Lambert MP, Paliwal A, Vaissiere T, Chemin I, Zoulim F, Tommasino M, Hainaut P, Sylla B, Scoazec JY, Tost J, Herceg Z. Aberrant DNA methylation distinguishes hepatocellular carcinoma associated with $\mathrm{HBV}$ and $\mathrm{HCV}$ infection and alcohol intake. J Hepatol. 2011; 54:705-15.

22. Nault JC. Pathogenesis of hepatocellular carcinoma according to aetiology. Best Pract Res Clin Gastroenterol. 2014; 28:937-47.

23. Tian YE, Xie XU, Lin Y, Tan G, Zhong WU. Androgen receptor in hepatocarcinogenesis: recent developments and perspectives. Oncol Lett. 2015; 9:1983-1988.

24. Lamarca A, Mendiola M, Barriuso J. Hepatocellular carcinoma: exploring the impact of ethnicity on molecular biology. Crit Rev Oncol Hematol. 2016; 102:111-7.

25. Llovet JM, Zucman Rossi J, Pikarsky E, Sangro B, Schwartz M, Sherman M, Gores G. Hepatocellular carcinoma. Nat Rev Dis Primers. 2016; 2:16018. 